\title{
Stochastic Resonance Based on Quantum Genetic Algorithm and Applications in Weak Signal Detection
}

\author{
Jing Zhe ${ }^{1, \text { a }}$, Guo Li ${ }^{2, b}$, Ruigang Zhang ${ }^{3, c}$ \\ ${ }^{1}$ Department of Missiles, Ordnance Engineering College, Shijiazhuang, 050003, China \\ ${ }^{2}$ Department of Missiles, Ordnance Engineering College, Shijiazhuang, 050003, China \\ ${ }^{3}$ Teaching and Research Section, Artillery Training Base of General Staff, Zhangjiakou, 050003, \\ China \\ aemail: jingzhe_happy@163.com, bemail:gl_ma@163.com, email:zhangruiganghappy@126.com
}

Keywords: Quantum Genetic Algorithm(QGA); Adaptive Stochastic Resonance(ASR); Weak Signal Detection

\begin{abstract}
Stochastic resonance(SR) is one of the applications in weak signal detection. Many researchers used the combination of the traditional first-order bistable system and genetic algorithm(GA), but the output of first-order bistable system is not smoothly enough, genetic algorithm has the phenomenon of losting in locally value. Aimming at the drawbacks above, second-order and under-damped stochastic resonance based on quantum genetic algorithm(QGA) is proposed. The test in simulation data and vibreation signals show that the presented method can raise the SNR ratio effectively, which has advantages of simplicity and fast convergence speed.
\end{abstract}

\section{Introduction}

The Italian scholar Benzi.R raised the stochastic resonance(SR) in 1981, which has become one of hot spots in detecting weak signals after 30 years of development. Being different from the other denoising methods, SR makes full use of noise to make some of its energy into the signal's in order to achieve the purpose of noise reduction. The occurrence of Adaptive stochastic resonance(ASR) is a milestone in the development of SR. ASR does not need prior knowledge of signal and noise, it makes the system reach SR and detect weak signals by adjusting system parameters.

In previous studies on ASR, scholars often used genetic algorithm(GA) for signal processing. If chose, crossover and mutation of GA were chosen improperly, GA would be more iterations, convergence would be slowly, easily fall into local minima and some other phenomena would come along, when SR parameters were optimized. Second-order and under-damped SR system is proposed [1], which characteristics are superior to the traditional model of the first-order in both the time domain and frequency domain. Quantum genetic algorithm(QGA) is a genetic algorithm based on the principles of quantum computing, which has been widely used in various fields[2], but there is little literature of its application on SR. QGA expresses quantum state vectors in the genetic code, with the use of quantum logic gates chromosome evolution in order to achieve better effective than conventional GA.

Deficiency of existing methods of SR in the presence of weak signal detection, this paper will propose a new method for detecting weak signal by combining the QGA and second-order and under-damped bistable SR system. In this paper, second-order and under-damped bistable SR system is the target to study on, the globally optimization capabilities of QGA is the method, and the output signal-to-noise ratio of SR system is the fitness objection for the system's parameters optimizing, thus to realize the detection of weak signal.

\section{Second-order and under-damper bistable SR system}

The effect of combining nose and periodic signals can cause SR phenomenon. From the viewpoint of mathematics and in terms of signal processing, it will enhance the low frequency and filter out high frequency signals at the same time, when the order of SR system increases. A 
second-order SR system is proposed, and its corresponding dynamic equation can be considered as follows:

$$
\frac{d^{2} x}{d t^{2}}+\gamma \frac{d x}{d t}=a x-b x^{3}+A \cos \left(2 \pi f_{0} t\right)+\zeta(t)
$$

where $x(t)$ denotes the system output, parameters $a$ and $b$ are positive real numbers, $\gamma$ is a damping parameter, $A \cos \left(2 \pi f_{0} t\right)$ is a periodic input signal with the amplitude $A$ and the frequency $f_{0}$, and $\zeta(t)$ is a Gaussian white noise with zero mean and $D$ intensity, and the noise is abided by the follows: $<\zeta(t), \zeta(0)>=2 D \delta(t)$.

In order to obtain the output signal, using the fourth-order Runge-Kutta for solving equations.

Signal-to-noise ratio(SNR) is one of the SR measures, and we obtain output SNR of the SR system by using power:

$$
\text { SNRout }=10 \lg \frac{P_{\text {signal }}}{P_{\text {noise }}}
$$

where $P_{\text {signal }}=\left|Y\left(k_{0}\right)\right|^{2}$ is an estimation of the power spectrum signal, $Y(k)$ is the Fourier transform of the output signal. $P_{\text {noise }}$ is the estimation of noise power spectrum.

\section{Quantum Genetic Algorithm with second-order and under-damped bistable SR system}

QGA is a stochastic search algorithm which combines GA and quantum computing theory. Compared with GA, QGA maintain a good population diversity and has a good global search capability.

The core of QGA is encoded qubits and quantum gates updates. In quantum computing, the qubit is a carrier of information, and a state of the quantum bit can be written as: $|\phi\rangle=\alpha|0\rangle+\beta|1\rangle$, where $|0\rangle,|1\rangle$ denote the spin-down and spin-up, $(\alpha, \beta)$ indicates that the corresponding state probability amplitude occurs two complex constants satisfy the following normalization condition: $|\alpha|^{2}+|\beta|^{2}=1$, where $|\alpha|^{2},|\beta|^{2}$ denote the probability of $|0\rangle,|1\rangle$. According to the characteristics of different problems, different evolutionary processes can be designed actuators, namely quantum gates. Under normal circumstances, quantum gate is often be used and its update process is as follows:

$$
\left[\begin{array}{c}
\alpha_{i}^{\prime} \\
\beta_{i}^{\prime}
\end{array}\right]=\left[\begin{array}{cc}
\cos \left(\theta_{i}\right) & -\sin \left(\theta_{i}\right) \\
\sin \left(\theta_{i}\right) & \cos \left(\theta_{i}\right)
\end{array}\right]\left[\begin{array}{c}
\alpha_{i} \\
\beta_{i}
\end{array}\right]
$$

where $\left(\alpha_{i}, \beta_{i}\right)^{T},\left(\alpha_{i}^{\prime}, \beta_{i}^{\prime}\right)^{T}$ denote the probability amplitude of the number of $i$ chromosome qubit rotation gate before and after the update, $\theta_{i}$ is the rotation angle.Make the system output SNR as the individual fitness function: $J=\operatorname{SNRout}(a, b, \gamma)$, optimize three parameters at the same time.

ASR based on QGA flow chart shown in Figure 1:

1) Initial population $Q\left(t_{0}\right)$, initial $\left(\alpha_{i}^{t}, \beta_{i}^{t}\right)$ as $(1 / \sqrt{2}, 1 / \sqrt{2})$, a chromosome is expressed probability of all possible state of superposition;

2) Put each parameters into second-order and under-damped bistable SR system and obtain the output SNR by forth-order Runge-Kutta;

3) Measured each individual of the initial population $Q\left(t_{0}\right)$, and obtain the corresponding solution $P(t)=\left\{p_{1}^{t}, p_{2}^{t}, \cdots, p_{n}^{t}\right\}$, where $p_{j}^{t}$ is the $j$ individual measurements in the $\mathrm{t}$ generation for the population;

4) Make the output SNR as the fitness function, evaluate each individual of $P(t)$, and record 
the best individual and the corresponding fitness;

5) Determine whether the optimization process could be completed, exit if it fix the conditions or continue the calculation;

6) Use the quantum gate to adjust the individual and obtain the new population; the number of iterations plus 1 , returning to step (5).

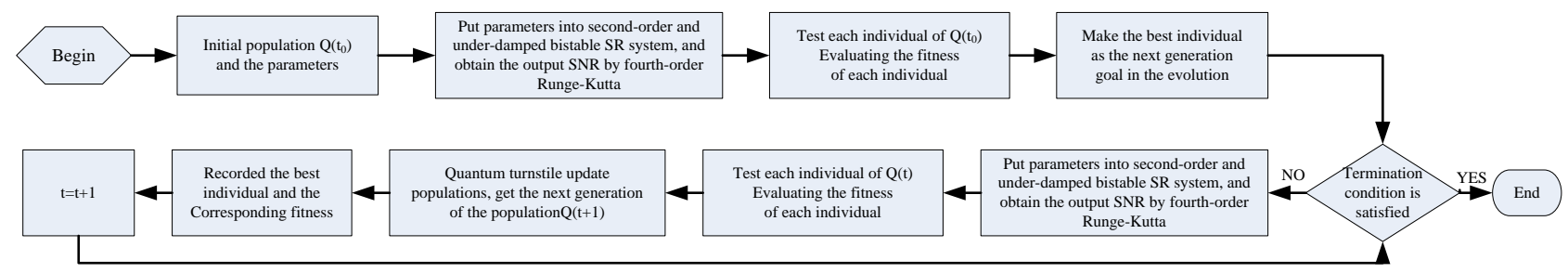

Fig.1. ASR based on QGA flow chart

\section{Simulation Signal Analysis}

Set the sampling frequency $5 \mathrm{~Hz}$, the sampling time is $500 \mathrm{~s}$, the sampling points is 2500; the amplitude is 0.1 and the frequency is $0.05 \mathrm{~Hz}$ of the input signal, and the noise intensity is 0.5 , then the input SNR is $-23.0103 \mathrm{~dB}$. Solve the Eq. (3) by fourth-order Runge-Kutta, and its step size $h$ is 0.2. In order to ensure the convergence of the solution process of SR, the parameters $a$ and $h$ should abide by the following relationship [3]: $a h \square 1$, then the parameters $a, b, \gamma$ search range of QGA is [0.01,5], [0.01,5] and [0.01,2] respectively. In QGA, the maximum of hereditary algebra is 300, the population size is 100 . First of all, optimizing of the parameters $a, b$ when let $\gamma=1$, then optimizing of the parameters $a, b, \gamma$ at the same time, and compare with GA(which the maximum of hereditary algebra and the population size is same to the QGA, gap, crossover and mutation probabilities are $0.95,0.7,0.05)$ and $a=1, b=1, \gamma=1$. Optimization results shown in Tabel 1:

Table 1. Optimization results

\begin{tabular}{|c|c|c|c|c|c|c|c|c|c|}
\hline & $a$ & $b$ & $r$ & $S N R_{\text {out }}$ & & $a$ & $b$ & $\mathrm{r}$ & $S N R_{\text {out }}$ \\
\hline 1 & 1 & 1 & 1 & -1.1241 & 3 & 0.061221 & 4.8579 & 1 & -0.2504 \\
\hline 2 GA & 1.17 & 0.51 & - & -0.6661 & 4 & 0.045845 & 4.8508 & 1.969 & -0.1176 \\
\hline
\end{tabular}

Tabel 1 shows that we obtain the maximum output SNR when optimizing the parameters $a, b, \gamma$ at the same time by QGA. The output SNR of QGA and second-order and under-damped bistable SR system is better than the one of GA and traditional SR system, when only optimize two parameters $a, b$.

The evolution of the QGA with optimizing three parameters and the GA with optimizing two parameters are shown as Fig. 2.

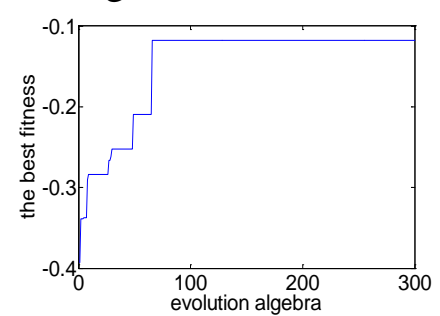

(a) QGA with optimizing three parameters

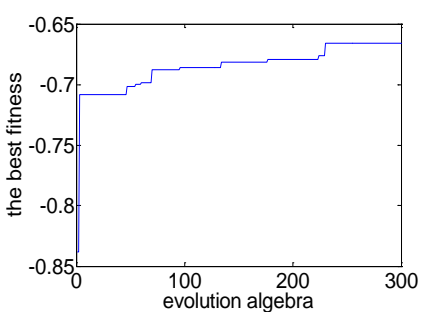

(b) QA with optimizing two parameters

Fig.2. Evolutionary process diagram

The Fig.2 shows that the termination algebra of the evolution of the optimal solution of QGA is 66, meanwhile the GA is 230. It obvious that QGA converges faster than GA.

The simulation is compared both GA with traditional SR and QGA with second-order and under-damped bistable SR system. The time domain waveform is shown in Fig. 3 and the frequency spectrum is shown in Fig. 4, respectively. 


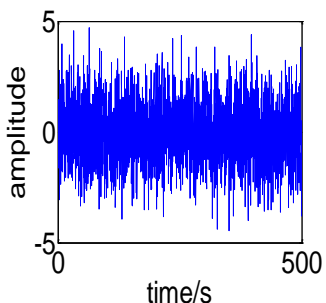

(a) original signal

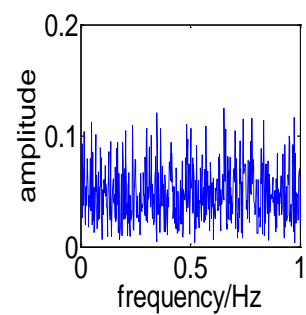

(a) original signal

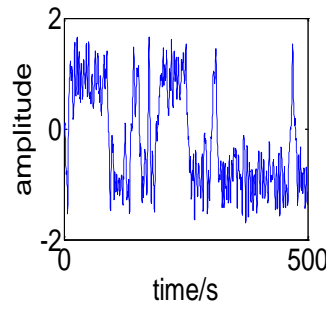

(b) 1

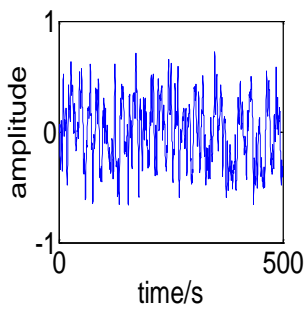

(c) 2

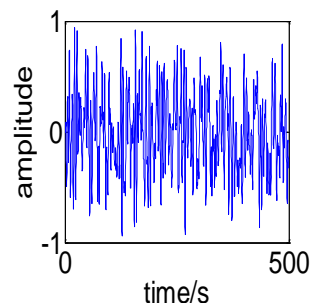

(d) 3

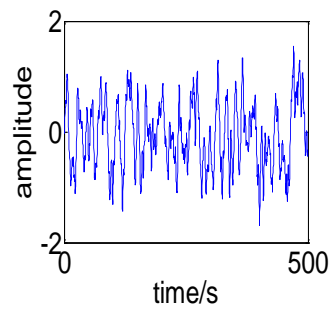

(e) 4

Fig.3. Time domain waveform

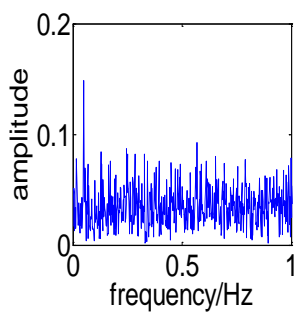

(b) 1

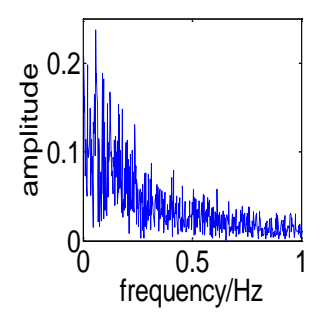

(c) 2

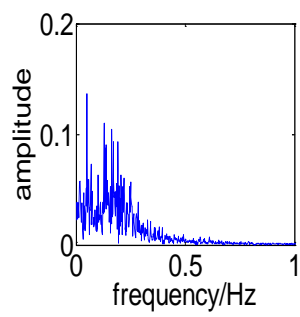

(d) 3

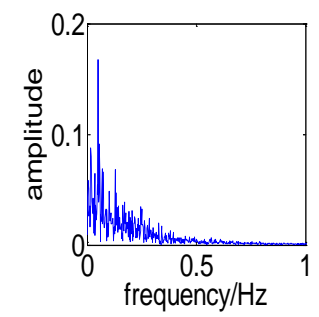

(e) 4

Fig.4. Frequency spectrum

Figure 3 is the time domain waveform of the input signal and the output signal by optimizing different parameters. Figure 3 (a) shows that the input signal is lost in the noise. Figure 3 (b) shows that the signal is distortion heavily when the parameters are not optimized. Figure 3 (c) and (d) indicates that portion of noise is removing with the effect of optimizing two parameters. When these three parameters were optimized at the same time, the effect of removing noise is better than optimizing two parameters.

Figure 4 is the frequency spectrum corresponding to Figure 3. The input signal frequency spectrum could be distinguished from the noise. The frequency could be obtained when the parameters without optimizing, and the frequency of noise is less when optimizing two parameters. Figure 4 (e) shows that the frequency of signal is clear and the frequency of noise is the least when optimizing three parameters at the same time.

Compared with the Figure 4 (c) and (e), it shows that the frequency of $0.05 \mathrm{~Hz}$ could be distinguished by the method of GA and traditional SR, but there are some other frequeny components which be more likely to be confused with the original frequency. Be different from above, the frequency of the signal which deal with the QGA and second-order and under-damped system is single and can be easily distinguished. It can be seen that the proposed method in this paper is more prominent in weak signal detection.

\section{Engineering Applications}

There are multiple pairs of friction in Swash-plate axial piston hydraulic pump and the friction between sliding-boot and swash-plate is the most complex one [4]. Therefore, The swash-plate wear is one of the most prone to failure in hydraulic pump. Its vibration signal is obtained easily and contains a wealth of diagnostic information. In this paper, the test device name and model number is shown in Table 2.

Table 2. The name and model number of experimental devices

\begin{tabular}{cc|cc}
\hline Test device name & Model number & Test device name & Model number \\
\hline hydraulic pump & SY-10MCY14-1EL & stable power supply & WYD-302B2 \\
drive motor & Y132M-4 & data logger & $\begin{array}{c}\text { DH-5920 dynamic signal } \\
\text { analyzer test }\end{array}$ \\
scceleration sensor & CA-YD-139 & & \\
\hline
\end{tabular}

The rated speed of drive motor is $1480 \mathrm{rp} / \mathrm{min}$, the number of hydraulic pump is 7 , the theoretical displacement is $10 \mathrm{ml} / \mathrm{r}$, the rated speed is $1500 \mathrm{rp} / \mathrm{min}$, the main relief valve pressure of hydraulic pump is $10 \mathrm{MPa}$, the sampling frequency is $20 \mathrm{kHz}$, sampling points is 5000 . The fundamental frequency of single plunger additional impact is $f=n / 60$ ( $\mathrm{n}$ is the motor speed), so the 
fundamental frequency in this test is $7 \times f=175 \mathrm{~Hz}$ due to the speed of the shaft is $1500 \mathrm{rp} / \mathrm{min}$. The faulty part replaced the normal one is shown as Figure 5. The time domain waveform and frequency spectrum of acquisition signal is shown as Figure 6.

Because of the adiabatic approximation theory, SR can only detect the signal with frequency much smaller than $1 \mathrm{~Hz}$. However, the frequency in this paper is $175 \mathrm{~Hz}$ which is much larger than $1 \mathrm{~Hz}$, we need scale transformation SR [5] to solve this problem. Firstly, collected signal is linear compressed by scale transformation, and then put the signal into the QGA and second-order and under-damped bistable SR system. At last, restore the measured data by compressing scale. In this paper, the compression multiples is 250, the range of parameters and QGA are the same as the simulation. The algorithm converges after 72 iterations by calculating. The optimal value of the parameter $a, b, \gamma$ is $0.1032,4.936$ and 0.095 respectively. The time domain waveform and the frequency spectrum of processing signal is shown as Figure 7.

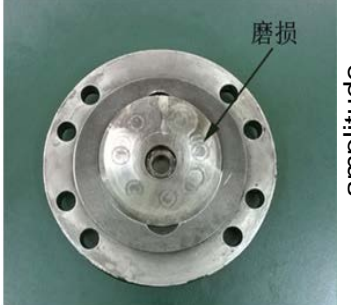

Fig.5. The failure part of swash-plate wear

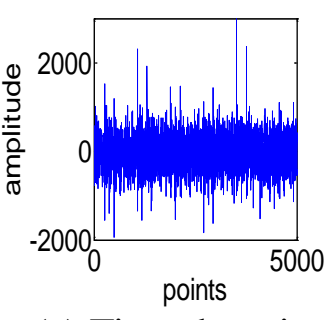

(a) Time domain waveform

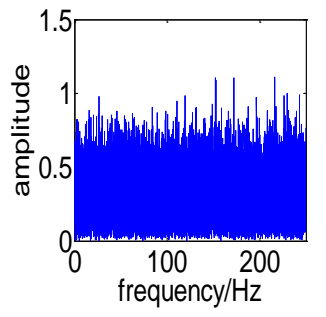

(b) Frequency spectrum

Fig.6. The time-frequency diagram

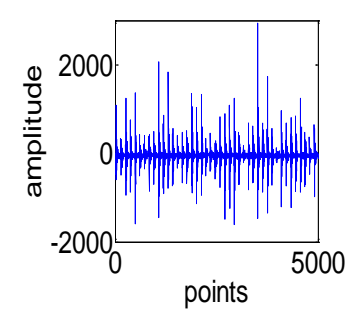

(a) Time domain waveform

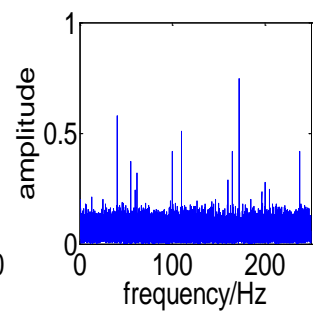

(b) Frequency spectrum

Fig.7. The time-frequency diagram The periodicity of processed signal is apparent in time domain and in frequency domain the detected frequency is $172 \mathrm{~Hz}$ which is similar to the shock vibration fundamental frequency of hydraulic pump.

\section{Conclusion}

This paper presents a new method of weak signal detection which combined QGA and second-order and under-damped bistable SR system. The method makes use of the good global search capability and diversity of the population of QGA, selects the output SNR as the individual fitness objective function, optimizes three parameters of second-order and under-damped bistable SR system at the same time and enables the extraction of weak signal in strong noise background.

\section{Acknowledgement}

In this paper, the research was sponsored by the National Natural Science Foundation of China under Grant (Project No. 51275524).

\section{References}

[1] Yong Xu, Juanjuan Li,et al. Levy noise-induced stochastic resonance in a bistable system [J]. The European Physical Journal B. 201386 198-204.

[2] Li Cheng, Li Fei. Improved quantum genetic algorithm and its application in FIR filter design [J]. Computer Engineering and Applications. 2009 45(4) 239-241.

[3] Xie Lei. Research of bearing vibration analysis and life evaluation methods [D]. Chengdu: University of Electronic Science and Technology of China. 2013.

[4] Zhang Changying, Gong Xiaoqun, et al. Measures to reduce wear and lower noise for swash-plate axial piston pump [J]. Chinese Hydraulics and Pneumatics. 201312 118-120.

[5] Fan Shengbo, Wang Taiyong, et al. Detection of weak periodic impact signal based on scale transformation stochastic resonance [J]. China Mechanical Engineering. 2006 17(4) 387-390. 